\title{
FUNCTIONS OF THE FIRST BAIRE CLASS WITH VALUES IN BANACH SPACES
}

\author{
CHARLES STEGALL \\ (Communicated by William J. Davis) \\ Dedicated to the memory of Zdenèk Frolik
}

\begin{abstract}
We characterize functions of the first Baire class with values in Banach spaces and give a short self-contained proof of a result more general than the following: If $T$ is a complete metric space, $X$ is a Banach space, and $\Phi: T \rightarrow \wp(X)$ (the power set of $X$ ) is a mapping that is usc in the weak topology then $\Phi$ has a selector of the first Baire class. This extends some results of Hansell, Jayne, Rogers, and Talagrand.
\end{abstract}

\section{INTRODUCTION}

These results concern multivalued functions from or into metric spaces, usually considered as subspaces of Banach spaces. We have not been able to summon much enthusiasm for noncomplete metric spaces, although some of our results seem to be valid for hereditarily Baire metrizable spaces, that is, metric spaces such that every closed subspace is a Baire space (satisfies the Baire category theorem). We cannot find our results, already more than a decade old, in the literature, but given the enormity of this literature (see [JR, HJT, H] for a beginning) it is quite possible that some of the results given here can be found elsewhere. Nonetheless, this paper is nearly self-contained and our proofs are so short and sweet, not to mention classical, that we present them to what, we are certain, will be an indulgent public. All of the techniques herein, if not all of the consequences of these techniques, with the exception of the elegant result of Frolík [F], of which we use such a special case that it is probably also classical, were known to us, Bourgain and others in 1976. In particular, we recall that the paper in [L] was pointed out to us by J. Hagler sometime before 1976. A function of the first Baire class, briefly said to be Baire one, is the pointwise limit of a sequence of continuous functions.

Received by the editors July 19, 1989 and, in revised forms, October 21, 1989 and April 30, 1990.

1980 Mathematics Subject Classification (1985 Revision). Primary 46B99; Secondary 26B05.

Key words and phrases. First Baire class, Banach spaces. 


\section{RESULTS}

In a metric space $T$ we denote by $\varrho(A, B)$ the distance between the subsets $A$ and $B$ of $T$, or, simply the distance if $A$ and $B$ are one point sets. A Hausdorff space is analytic if it is the continuous image of a complete separable metric space. A completely regular space is Čech complete if it is a $\mathrm{G}_{\delta}$ subset of its Stone-Čech compactification.

Theorem (Souslin, Kuratowski [K]). Let $M$ be analytic, metrizable and uncountable. Then $M$ contains a subspace homeomorphic to the Cantor space.

The theorem above (the only case that we require) is a special case of the result below, which also gives another way of looking at some model theoretic results of Mansfield (see [M, p. 79]). The weight of a topological space $T$, denoted by wt $T$, is the smallest cardinal $\kappa$ such that there exists a basis for the topology of cardinality $\kappa$. We denote by card $T$ the cardinality of $T$. We denote by res $T$ the resolution index of $T$ which is the the smallest cardinal $\lambda$ such that there exists a Čech complete space $S$ so that wt $S=\lambda$ and there exists a continuous surjection $f: S \rightarrow T$. Obviously, res $T \leq$ card $T$ because if we denote by $S$ the set $T$ with the discrete topology then $S$ is a complete metric space and, therefore, is Čech complete. A topological space $T$ is perfect if every nonempty open subset has more than one point; if $T$ is Hausdorff this means that every nonempty open subset of $T$ is infinite.

Theorem 1. Let $T$ be a Hausdorff space such that res $T<\operatorname{card} T$ then $T$ contains a compact perfect subset. Suppose that $f: S \rightarrow T$ is a continuous surjection from the Cech complete space $S$ and $\lambda=$ wt $S<\operatorname{card} T$. If either $S$ or $T$ is metrizable then $T$ contains the Cantor space.

Proof. Let $\mathbf{B}$ be a basis for the topology of $S$ such that card $\mathbf{B} \leq \lambda$. As in the Cantor-Bendixson Theorem, define

$$
\mathbf{V}=\{B \in \mathbf{B}: \text { card } f(B) \leq \lambda\}
$$

The union $V$ of all the sets in $\mathbf{V}$ has the property that $f(V)$ has cardinality no greater than $\lambda$ and any nonempty relatively open subset $W$ of $S \backslash V$ has the property that card $f(W)>\lambda$. Since $S \backslash V$ is closed and closed subsets of Čech complete spaces are Čech complete, we may assume that $V=\varnothing$. Let $\beta S \backslash S=\bigcup_{n} S_{n}$ where $\left\{S_{n}\right\}$ is an increasing sequence of compact subsets of $\beta S$. By induction, we construct, for each pair of integers $i$ and $j$ such that $0 \leq j<2^{i}$, nonempty open sets $V_{i, j} \subseteq S$ and $U_{i, j} \subseteq T$ such that

$$
\begin{aligned}
& f\left(V_{i, j}\right) \subseteq U_{i, j}, \\
& \left\{U_{i, j}: 0 \leq j<2^{i}\right\} \text { is pairwise disjoint for each } i, \\
& \bar{V}_{i+1,2 j} \cup \bar{V}_{i+1,2 j+1} \subseteq V_{i, j},
\end{aligned}
$$


and

$$
{\overline{V_{i, j}}}^{\beta S} \cap S_{i}=\varnothing \text { for all } i \text {. }
$$

Let $T=U_{0,0}$ and $S=V_{0,0}$ and suppose that we have made this construction up to order $i$. Fix $i$ and $j$. Since $f\left(V_{i, j}\right)$ is infinite, choose $s$ and $s^{\prime}$ in $V_{i, j}$ such that $f(s) \neq f\left(s^{\prime}\right)$. Choose $U_{i+1,2 j}$ and $U_{i+1,2 j+1}$ disjoint open sets such that $f(s) \in U_{i+1,2 j}, f\left(s^{\prime}\right) \in U_{i+1,2 j+1}$, and $U_{i+1,2 j} \cup U_{i+1,2 j+1} \subseteq U_{i, j}$. Since $f$ is continuous, choose open sets $V_{i+1,2 j}$ and $V_{i+1,2 j+1}$ containing, respectively, $s$ and $s^{\prime}$ so that $\bar{V}_{i+1,2 j} \cup \bar{V}_{i+1,2 j+1} \subseteq V_{i, j}, f\left(V_{i+1,2 j}\right) \subseteq U_{i+1,2 j}$, $f\left(V_{i+1,2 j+1}\right) \subseteq U_{i+1,2 j+1}$ and

$$
\left({\overline{V_{i+1,2 j}}}^{\beta S} \bigcup{\overline{V_{i+1,2 j+1}}}^{\beta S}\right) \cap S_{i+1}=\varnothing .
$$

Observe that

$$
K_{0}=\bigcap_{0 \leq i<\infty}\left(\bigcup_{0 \leq j<2^{i}}{\overline{V_{i, j}}}^{\beta S}\right) \subseteq \beta S \backslash\left(\bigcup_{0 \leq i<\infty} S_{i}\right)=S .
$$

Thus, $K_{0}$ is compact as well as each $K_{i, j}=K_{0} \cap V_{i, j}$. Choose a maximal decreasing filter $\mathbf{F}$ so that

$$
\mathbf{F} \subseteq\left\{E \subseteq K_{0}: E \text { is closed and } E \cap K_{i, j} \neq \varnothing \text { for all } i \text { and } j\right\} .
$$

It follows that $\mathbf{F}$ has a minimal element $K=\bigcap_{E \in \mathbf{F}} E$. If $W$ is open and $W \cap K \neq \varnothing$ then $K \backslash W \notin \mathbf{F}$ which means that there exists $K_{i, j}$ such that $(K \backslash W) \cap K_{i, j}=\varnothing$, which means that $W \cap K \cap K_{i, j} \supseteq K \cap K_{i, j}$ and $W \cap K \cap K_{k, l} \neq \varnothing$ for all $K_{k, l} \subseteq K_{i, j}$. Thus, $K$ is perfect and $f(K)$ is perfect because $f(W \cap K) \cap U_{k, l} \neq \varnothing$ for all $U_{k, l} \subseteq U_{i, j}$. If $S$ is metrizable and if we assume in addition that the diameter of $V_{i, j}$ is less than $2^{-n}$ then $K_{0}$ is homeomorphic to the Cantor space and $f$ restricted to $K_{0}$ is a homeomorphism. If $T$ is metrizable and if we assume in addition that the diameter of $U_{i, j}$ is less than $2^{-n}$ then $f\left(K_{0}\right)$ is homeomorphic to the Cantor space.

The following result is a special case of a result due to Frolík [F] (see also [S1]).

Theorem 2. Suppose that $T$ is a complete separable metric space, $M$ is metric and $f: T \rightarrow M$ is a function such that $g \circ f$ has an analytic graph for every continuous $g: M \rightarrow \Re$. Then $f(T)$ is separable.

Proof (in [F]). Suppose that $f(T)$ is not separable. Choose $D \subseteq f(T)$, uncountable, so that the distance between any two points of $D$ is more than $\delta>0$. Assume that the cardinality of $D$ is no greater than that of $[0,1]$. Let $g_{0}: D \rightarrow[0,1]$ be any one to one function. Since $D$ is discrete it is closed, $g_{0}$ is continuous, and by the Tietze-Urysohn Theorem, we may extend $g_{0}$ to a continuous $g: M \rightarrow[0,1]$. By assumption, $g \circ f$ has an analytic graph. Also, for any $E \subseteq D$, the distance function $\varrho(\cdot, E)$ is continuous. 
Thus, $f^{-1}(E)=\{t: \varrho(f(t), E)=0\}$ is analytic. Since $g$ is one to one on $D$ it follows that every subset of $g(D)$ is analytic. From Souslin's Theorem $g(D)$ contains a copy of the Cantor set, but the Cantor set has non analytic subsets, which is a contradiction.

We repeat (see [L]) a few elementary observations concerning functions of the first Baire class with values in a Banach space $Z$. If $g: T \rightarrow Z$ is Baire one and $h: T \rightarrow \Re$ is Baire one then the product $h g$ is Baire one. If $U$ is an open subset of $T$ then $\chi_{U}: T \rightarrow \Re$ is not only in the first Baire class but if we define $h_{n}: T \rightarrow \Re$ by

$$
h_{n}(t)=\left(1 \wedge\left(0 \vee\left(\varrho(t, T \backslash U)-\frac{1}{n}\right)\right)\right)^{1 / n}
$$

then $\chi_{U}: T \rightarrow \Re$ is the pointwise limit of the continuous functions $\left\{h_{n}\right\}$, each of which vanishes on a neighborhood of $T \backslash U$, and $0 \leq h_{n} \leq \chi_{U}$ for all $n \in \mathscr{N}$. If $\left\{h_{n}\right\}$ is any sequence of Baire one functions such that $\sum_{n}\left\|h_{n}\right\|<+\infty$ where $\left\|h_{n}\right\|=\sup _{t \in T}\left\|h_{n}(t)\right\|$ then $\sum_{n} h_{n}$ is Baire one. Suppose that $f: T \rightarrow Z$ is a function such that for every $\epsilon>0$ there exists a Baire one function $g: T \rightarrow Z$ such that $\|f(t)-g(t)\|<\epsilon$ for all $t \in T$; then $f$ is in the first Baire class. To see this choose $f_{n}$ Baire one so that $\left\|f_{n}-f\right\|<2^{-n-1}$ and choose continuous functions $\left\{f_{n, i}: n, i \in \mathscr{N}\right\}$ so that

$f_{1, i} \rightarrow f_{1}$ pointwise and for $n \geq 2$,

$f_{n, i} \rightarrow f_{n+1}-f_{n}$ pointwise and $\left\|f_{n, i}\right\|<2^{-n}$.

The desired continuous functions are $g_{i}=\sum_{n} f_{n, i}$.

For real valued functions, proofs were given, independently, by Bourgain [B], the author [S2] and others before 1976. Indeed, the ideas in [L] only require modernization to work in this case; the set $\mathbf{U}_{\epsilon}$ considered below appears in [L]. Only a change in notation is required so that everything works in the multivalued case. Certainly, this proof is classical in the case that $T$ is separable; replacing Lindelöf arguments in [L] by paracompactness arguments is an obvious device.

Theorem 3. Suppose that $T$ is a complete metric space, $Z$ is a Banach space and $\Phi: T \rightarrow \wp(Z)$ is a multivalued map with the following property:

for each $\epsilon>0$, each nonempty and closed subset $C$ of $T$ and each closed ball $B$ of $Z$ with perhaps infinite radius such that $\Phi(t) \cap B \neq \varnothing$ for all $t \in C$, there exist open subsets $U \subseteq T$ and $V \subseteq Z$ such that $U \cap C \neq \varnothing, V \cap B \neq \varnothing$, the diameter of $V$ is less than $\epsilon$, and $\Phi(t) \cap V \neq \varnothing$ for all $t \in U \cap C$.

Then there exists a Baire one function $f: T \rightarrow Z$ such that $\varrho(f(t), \Phi(t))=0$ for all $t \in T$.

Proof. For a fixed positive $\epsilon$ define the following:

$$
\begin{aligned}
\mathbf{U}_{\epsilon}=\{U \subseteq Z: U \text { is open and there exists a Baire one } \\
\\
\left.f_{U}: T \rightarrow Z \text { so that } \varrho\left(f_{U}(t), \Phi(t)\right) \leq \epsilon \text { for all } t \in U\right\} .
\end{aligned}
$$


We shall show that $U_{0}=\bigcup\left\{U \in \mathbf{U}_{\epsilon}\right\}$ is in $\mathbf{U}_{\epsilon}$ and that $U_{0}=T$. Since the metric space $U_{0}$ is paracompact there exists a locally finite collection $\left\{V_{\gamma}: \gamma \in\right.$ $\Gamma\} \subseteq \mathbf{U}_{\epsilon}$ such that $\bigcup\left\{V_{y}: \gamma \in \Gamma\right\}=U_{0}$. Assume that $\Gamma$ is well ordered with minimal element 1 . Define $V_{0}=\varnothing$ and for each $\gamma \in \Gamma$ choose a sequence of continuous functions $r_{\gamma, n}: T \rightarrow \Re$ such that each $r_{\gamma, n}$ is supported on $V_{\gamma}$ and

$$
r_{\gamma, n} \rightarrow \chi_{V_{\gamma} \backslash\left(\cup_{\beta<\gamma} V_{\beta}\right)}
$$

pointwise. Choose continuous functions $f_{\gamma, n}: T \rightarrow Z$ so that

$$
f_{\gamma, n} \rightarrow f_{V_{y}} .
$$

Choose continuous functions $s_{n}: T \rightarrow \Re$ so that $s_{n} \rightarrow \chi_{U_{0}}$ and $s_{n}(t)=0$ if $\varrho\left(t, T \backslash U_{0}\right) \leq \frac{1}{n}$. Define

$$
g_{n}(t)=s_{n}(t) \sum_{\gamma} r_{\gamma, n}(t) f_{\gamma, n}(t)
$$

Since $\left\{V_{\gamma}: \gamma \in \Gamma\right\}$ is locally finite on $U_{0}$, each $g_{n}$ is well defined and continuous on all of $T$. It is routine to check that

$$
f(t)=\lim _{n} g_{n}(t)=\chi_{U_{0}}(t) \sum_{\gamma} \chi_{V_{\gamma} \backslash \cup_{\beta<\gamma} U_{\beta}}(t) f_{V_{\gamma}}(t)
$$

is Baire one and $\varrho(f(t), \Phi(t)) \leq \epsilon$ if $t \in U_{0}$. If $T \backslash U_{0} \neq \varnothing$ choose $z_{0} \in Z$ and $U$ open such that $\Phi(t) \cap B\left(z_{0}, \epsilon\right) \neq \varnothing$ for all $t \in U \cap\left(T \backslash U_{0}\right)$. Clearly, $U \cup U_{0}$ is in $\mathbf{U}_{\epsilon}$ because $f+\chi_{U \backslash U_{0}} z_{0}$ is Baire one. Thus, $T$ is in $\mathbf{U}_{\epsilon}$. If $\Phi$ is single valued then we are finished. Suppose that $\Phi$ satisfies the hypothesis above, $r>$ 0 , and $f: T \rightarrow Z$ is a Baire one function such that $(\Phi(t)-f(t)) \cap B(0, r) \neq \varnothing$ for all $t \in T$. Then for any $\delta>0$ there exists a Baire one function $h: T \rightarrow Z$ such that $\|h\| \leq r+\delta$ and

$$
(\Phi(t)-f(t)-h(t)) \cap B(0, \delta) \neq \varnothing
$$

for all $t \in T$. The proof of this is the same as above. Observe that for a fixed $z_{0} \in Z$ the translated relation $\Phi-z_{0}$ also satisfies the hypothesis of the theorem. Suppose that we have an open $U \subseteq T$ and $g: T \rightarrow B(0, r+\delta)$ that is Baire one and

$$
(\Phi(t)-f(t)-g(t)) \cap B(0, \delta) \neq \varnothing
$$

for all $t \in U$. Suppose that $C=T \backslash U \neq \varnothing$. Since $f \mid C$ has a point of continuity there exist $z_{0} \in Z$ and $U_{1}$ such that $U_{1} \cap C \neq \varnothing$ and $f\left(\overline{C \cap U_{1}}\right) \subseteq$ $B\left(z_{0}, \delta / 2\right)$. Let $D=\overline{C \cap U_{1}}$. Observe that for all $t \in D$ we have that

$$
\left(\Phi(t)-z_{0}\right) \cap B\left(0, r+\frac{\delta}{2}\right) \neq \varnothing .
$$

There exist $U_{2}$ and $w \in Z$ such that $B(w, \delta / 4) \cap B(0, r+\delta / 2) \neq \varnothing$, $U_{2} \cap D \neq \varnothing$ and $\left(\Phi(t)-z_{0}\right) \cap B(w, \delta / 4) \neq \varnothing$ for all $t \in U_{2} \cap D$. Observe that $W=U_{2} \cap U_{1} \cap C \neq \varnothing$. Let

$$
h=\chi_{U} g+\chi_{W}\left(w-z_{0}\right) .
$$


Continuing, we may construct a sequence of Baire one functions $\left\{f_{n}\right\}$ such that $\varrho\left(f(t)+\sum_{i \leq n} f_{i}(t), \Phi(t)\right) \leq 1 / 2^{n}$ for all $n$ and $\left\|f_{n}\right\|<3 / 2^{n}$ for all $n \geq 2$.

Recall that a metric space $T$ isometrically embeds in a Banach space. Fix $t_{0} \in T$ and define $f: T \rightarrow C_{b}(T)$ by $f(t)=\varrho(\cdot, t)-\varrho\left(\cdot, t_{0}\right)$, where $C_{b}(T)$ is the Banach algebra of bounded continuous functions on $T$. With this recollection the reader should make the obvious interpretations of the following theorem if one only assumes that $Z$ is a metric space. If $Z$ is the space of real numbers and $T$ is separable the following is in Hausdorff's book [Ha] (compare [L]). Notice that neither the theorem above nor the one below requires the Dugundji extension theorem. In fact, we have shown that if $S$ is a closed subspace of the complete metric space $T$ and $f: S \rightarrow Z$ is a Baire one function into the Banach space $Z$ then $g: T \rightarrow Z$, defined to be $f$ on $S$ and the origin on $T \backslash S$, is Baire one. The results of [B] also yield the equivalence of (i)-(iii) below. Since a Fréchet linear space embeds linearly as a closed subspace of a countable product of Banach spaces the following also holds when $Z$ is a Fréchet linear space. The proof actually obtains continuous functions whose images are in the norm space generated by the image of $\Phi$.

Theorem 4. Let $f: T \rightarrow Z$ be a function from the complete metric space $T$ to the Banach space $Z$. The following are equivalent:

(i) $f$ is in the first Baire class;

(ii) $f \mid C$ has a point of continuity for each closed subset $C$ of $T$;

(iii) $f \mid C$ has a point of continuity for each compact subset $C$ of $T$;

(iv) $h \circ f$ is in the first Baire class for each continuous function $h: Z \rightarrow \Re$;

(v) $f^{-1}(C)$ is $G_{\delta}$ for each closed subset $C$ of $Z$.

Proof. Condition (i) implies all of the others; these are, of course, results of Baire rephrased (see [L]). Suppose that we have (iii). Suppose that $C$ is a closed subset of $T$ such that $f \mid C$ has no point of continuity. For notational convenience, assume that $T=C$. There exists $\epsilon>0$ such that

$$
\left\{t \in T: \limsup _{s \rightarrow t}\|f(t)-f(s)\| \geq \epsilon\right\}
$$

contains a nonempty open set $U$. Choose an open $V \subseteq U$ and $\delta>0$ so that $\varrho(V, T \backslash U)>\delta$. Choose $t_{1} \in V$ and let $F_{1}=\left\{t_{1}\right\}$. Given $F_{n}$ we define $F_{n+1}$ as follows: for each $t \in F_{n}$ choose $s_{t} \in T, \varrho\left(t, s_{t}\right)<2^{-n} \delta$, and $\left\|f(t)-f\left(s_{t}\right)\right\|>\frac{\epsilon}{2}$. Define $F_{n+1}=F_{n} \cup\left\{s_{t}: t \in F_{n}\right\}$ and

$$
F=\overline{\bigcup_{n} F_{n}} \text {. }
$$

It is routine to check that $F$ is compact and $f \mid F$ has no point of continuity. Using Theorem 3, we have that (i), (ii), and (iii) are equivalent. If $f$ is in the first Baire class, $\left\{f_{n}\right\}$ is a sequence of continuous functions that converge 
pointwise to $f$ and $C$ is a closed subset of $Z$ then

$$
f^{-1}(C)=\bigcap_{m=1}^{\infty} \bigcap_{k=1}^{\infty} \bigcup_{n=k}^{\infty}\left\{t: \varrho\left(f_{n}(t), C\right)<\frac{1}{m}\right\}
$$

is $\mathrm{G}_{\delta}$. It only remains to show that each of (iv) and (v) implies (iii). Let $K$ be any compact subset of $T$. Observe that if we have (v) and $h: Z \rightarrow \Re$ is continuous then $(h \circ f) \mid K$ is in the first Baire class (this is very easy, and is explicitly stated in $[\mathrm{H}]$ ) and using the equivalence of (i)-(iii) we have that $h \circ f$ is Baire one. Thus, (v) implies (iv). Also, if we have (iv) then Theorem 2 says that $f(K)$ is a separable subset of $Z$. Choose a dense sequence $\left\{z_{i}\right\}$ in $f(K)$. Choose a point $t \in K$ such that each function $g_{i}: K \rightarrow \Re$ defined by $g_{i}(t)=\left\|f(t)-z_{i}\right\|$ is continuous at $t$. It is easy to check that $f \mid K$ is continuous at $t$.

Suppose that $T$ is a regular space that contains a dense strongly countably complete space (see [F2]), or, equivalently (this is not completely trivial [S1]), a regular space that contains a dense subspace that has a strategy in the sense of [Ch]. Such spaces are characterized by the following properties: there exist $S \subseteq T$ that is dense and a sequence of open families $\left\{\mathbf{V}_{n}\right\}$ such that each $\mathbf{V}_{n}$ covers $S$ and, if $\left\{s_{n}\right\} \subseteq S$ is a sequence such that for all $n \in \mathscr{N}$ there exists $V_{n} \in \mathbf{V}_{n}$ such that $\left\{s_{m}: n \leq m\right\} \subseteq V_{n}$, then $\left\{s_{n}\right\}$ has a cluster point. Notice that this property is inherited by open subsets. Such spaces are Baire spaces and for want of a better name we shall call them complete Baire spaces. The following, in the single valued case, gives a very easy proof to the main result of [N] and, in the multivalued case, shows that compactness conditions in [Ch] are not necessary. This proof does not seem to work in the more general setting of [S4].

Theorem 5. Let $T$ be a complete Baire space, $Z$ a Banach space and $\Phi: T \rightarrow$ $\wp(Z)$ taking nonempty values such that

$$
\{t \in T: \Phi(t) \cap D \neq \varnothing\}
$$

is a closed subset of $T$ for each norm closed, bounded and convex subset $D$ of $Z$. Then there exists a (single valued) function $h: T \rightarrow Z$ such that $\varrho(h(t), \Phi(t))=0$ for all $t \in T$ and $h$ is norm continuous at each point of a dense $G_{\delta}$ subset of $T$.

Proof. We shall show that for any $\epsilon>0$ there exist nonempty open sets $U \subseteq T$ and $V \subseteq Z$ so that the diameter of $V$ is less than $\epsilon$ and $\Phi(t) \cap V \neq \varnothing$ for all $t \in U$. Since $T$ is a Baire space we may assume that $\Phi(t) \cap B(0, r) \neq \varnothing$ for all $t \in T$ and for some $r>0$. Indeed, we may assume that $\Phi(t) \subseteq B(0, r)$ for all $t \in T$. Suppose that we have $\epsilon>0$ such that

$$
\{t \in T: \Phi(t) \cap B(z, \epsilon) \neq \varnothing\}
$$

is nowhere dense (first category is enough) for all $z \in Z$. Fix $t_{1} \in T$ and $z_{1} \in \Phi\left(t_{1}\right)$. Choose any open set $U_{1}$ containing $t_{1}$. The Baire category theorem 
and a simple compactness argument shows that there exists a small open set $U_{2}$ so that $\overline{U_{2}} \subseteq U_{1}$ and

$$
\varrho\left(\Phi\left(\overline{U_{2}}\right),\left[\left\{z_{1}\right\}\right] \cap B(0, r)\right) \geq \epsilon / 2,
$$

where $\left[\left\{z_{1}\right\}\right]$ denotes the smallest norm closed linear subspace containing $\left\{z_{1}\right\}$. Choose any $t_{2} \in U_{2}$ and in general, we choose a sequence of open sets $\left\{U_{n}\right\}$, a sequence of points $\left\{t_{n}\right\}$ in $T$, and a sequence of vectors $\left\{z_{n}\right\}$ in $Z$ so that

$$
\begin{gathered}
t_{n} \in \overline{U_{n}} \subseteq U_{n-1}, \\
z_{n} \in \Phi\left(t_{n}\right),
\end{gathered}
$$

and

$$
\varrho\left(\Phi\left(\overline{U_{n}}\right),\left[\left\{z_{1}, \ldots, z_{n-1}\right\}\right] \cap B(0, r)\right) \geq \epsilon / 2 .
$$

If $\overline{U_{n}} \subseteq V_{n}$ for some $V_{n} \in \mathbf{V}_{n}$ then $\left\{t_{n}\right\}$ has a cluster point $t$ and since $\left[\left\{z_{n}\right\}\right] \cap B(0, r)$ is closed and convex it follows that $\Phi(t) \cap\left[\left\{z_{n}\right\}\right] \cap B(0, r) \neq \varnothing$ which is a contradiction. The remainder of the proof only requires slight modification of the techniques already given here (see also Chapter 6 of [S1]).

Let $\left\{\mathbf{W}_{n}\right\}$ be a sequence of families of open sets maximal with respect to being pairwise disjoint, $\mathbf{W}_{n}$ is subordinate to $\mathbf{V}_{n}, \mathbf{W}_{n+1}$ is subordinate to $\mathbf{W}_{n}$, and if $W \subseteq W^{\prime}, W \in \mathbf{W}_{n+1}$, and $W^{\prime} \subseteq \mathbf{W}_{n}$, then there exist closed balls $F$ and $F^{\prime}$ in $Z$ so that $F \cap F^{\prime} \neq \varnothing$, the diameter of $F$ is no more than $2^{-n-1}$, the diameter of $F^{\prime}$ is no more than $2^{-n}, \Phi(t) \cap F^{\prime} \neq \varnothing$ if $t \in W^{\prime}$, and $\Phi(t) \cap F \neq \varnothing$ if $t \in W$. Define $h_{1}: T \rightarrow Z$ so that $h_{1}$ is constant on each $W \in \mathbf{W}_{1}, \varrho\left(h_{1}(t), \Phi(t)\right) \leq \frac{1}{2}$ for $t$ in some $W \in \mathbf{W}_{1}$ and $h_{1}(t) \in \Phi(t)$ if $t \notin \bigcup \mathbf{W}_{2}$. Given $h_{n-1}$ define $h_{n}: T \rightarrow Z$ so that $h_{n}$ is constant on each $W \in \mathbf{W}_{n}, \varrho\left(h_{n}(t), \Phi(t)\right) \leq 2^{-n}$ for $t$ in some $W \in \mathbf{W}_{n+1}, h_{n}(t)=h_{n-1}(t)$ if $t \in\left(\bigcup \mathbf{W}_{n}\right) \backslash\left(\bigcup \mathbf{W}_{n+1}\right)$ and $\varrho\left(h_{n}(t), h_{n-1}(t)\right) \leq 2^{-n+2}$ if $t \in \bigcup \mathbf{W}_{n+1}$. Clearly, $\lim h_{n}(t)=h(t)$ exists for all $t \in T$ and $h$ is norm continuous at each point of $\cap \bigcup \mathbf{W}_{n}$ and $\varrho(h(t), \Phi(t))=0$ for all $t \in T$. Each $\bigcup \mathbf{W}_{n}$ is dense in $T$.

Theorem 6. Let $T$ be a complete metric space, $Z$ a Banach space and $\Phi: T \rightarrow$ $\wp(Z)$ taking nonempty values such that

$$
\{t \in T: \Phi(t) \cap D \neq \varnothing\}
$$

is a closed subset of $T$ for any norm closed, bounded, and convex subset $D$ of $Z$. Then there exists a Baire one function $f: T \rightarrow Z$ such that $\varrho(f(t), \Phi(t))=0$ for all $t \in T$.

Proof. The proof of Theorem 5 says that $\Phi$ satisfies the hypothesis of Theorem 3.

Obviously, the hypothesis of Theorem 5 is satisfied if $\Phi$ is single valued and weakly continuous. If $Z$ is the Banach space $C(K)$ of continuous functions on the compact space $K$ then we need only assume that

$$
\{t \in T: \Phi(t) \cap D \neq \varnothing\}
$$


is a closed subset of $T$ for each simply closed, norm bounded, and convex subset $D$ of $Z$. We need only replace each $\left[\left\{z_{1}, \ldots, z_{n}\right\}\right]$ in Theorem 5 by the finite dimensional subspace of $C(K)$ spanned by all polynomials of degree less than $n+1$ with variables $\left\{z_{1}, \ldots, z_{n}\right\}$. This generalizes the results of [HJT].

Corollary 7. Let $\mathbf{F}$ be a family of functions, each of which satisfies ( $\mathrm{v}$ ) of Theorem 4 , from the complete metric space $T$ to the metric space $Z$. If $\mathbf{F}$ is countably compact in the simple topology then any cluster point of $\mathbf{F}$ is Baire one. If $\mathbf{F}$ is countably compact and $T$ is separable then $\mathbf{F}$ is compact in the simple topology. Indeed, $\mathbf{F}$ is angelic (see [BFT] and [S1]).

Proof. We may assume that $Z$ is a Banach space and it follows that $\mathbf{F}$ is a subset of the first Baire class. Suppose that $f$ is a cluster point of $\mathbf{F}$. If $\mathbf{F}$ is countably compact, $h: Z \rightarrow \Re$ is continuous and $K \subseteq T$ is compact then $\{(h \circ g) \mid K: g \in \mathbf{F}\}$ is a countably compact set of functions on $K$. From [BFT] and the results above it follows that $f$ is Baire one. Suppose in addition, that $T$ is separable; we must show that if $\mathbf{F}$ is countably compact then it is compact. Let $f: T \rightarrow Z$ be a cluster point of $\mathbf{F}$ and let $h: Z \rightarrow \Re$ be a continuous function. From Theorem 4 it follows that $f$ is Baire one and has separable range. Suppose that $S$ is a separable and closed subspace of $Z$ that contains $f(T)$. Define for each $g \in \mathbf{F}$

$$
\tilde{g}: T \times S \rightarrow \Re
$$

by $\tilde{g}(t, s)=\|g(t)-s\|$ and $\tilde{f}$ in an analogous manner. Clearly, $\{\tilde{g}: g \in \mathbf{F}\}$ is a countably compact set of real valued Baire one functions on the complete separable metric space $T \times S$. Apply the results of [BFT] again and we have that $\tilde{f}$ is the pointwise limit of a sequence in $\{\tilde{g}: g \in \mathbf{F}\}$. It follows easily that $f$ is the pointwise limit of a sequence in $\mathbf{F}$. Secondly, we must show that if $\mathbf{F}$ is compact in the simple topology and $\mathbf{G} \subseteq \mathbf{F}$ then any cluster point of $\mathbf{G}$ is the limit of a sequence in $\mathbf{G}$ and this is done in an analogous manner.

The following result is in [HJT] and in [JR]. It follows immediately from properties of dentability and the results above.

Theorem 8. Suppose that $T$ is a complete metric space, $X$ is a Banach space and $Y \subseteq X^{*}$ is norming and such that every norm bounded subset of $X$ is $Y$ dentable. Then, for every $\Phi: T \rightarrow \wp(X)$ that is $\sigma(X, Y)$ upper-semicontinuous with each $\Phi(t)$ a nonempty $\sigma(X, Y)$ compact set, there exists a Baire one function $f: T \rightarrow X$ in the norm topology such that $\varrho(f(t), \Phi(t))=0$ for all $t \in T$.

Corollary 9. Suppose that $Z$ is a Banach space. Then $Z^{*}$ has the RadonNikodym property if and only if there exists a function $f: Z \rightarrow Z^{*}$ that is Baire one with respect to the norm topologies and $\langle z, f(z)\rangle=\|z\|$ for all $z \in Z$. Proof. If $Z^{*}$ has the Radon-Nikodým property then the the function $\Phi: Z \rightarrow$ $\wp\left(Z^{*}\right)$ defined by $\Phi(z)=\left\{z^{*}:\left\|z^{*}\right\| \leq 1\right.$ and $\left.\left\langle z, z^{*}\right\rangle=\|z\|\right\}$ satisfies 
Theorem 3, which is a consequence of the weak* small slices property of $Z^{*}$ [S3]. The other direction is much more difficult than any of the results presented here, in that it depends on a fundamental result of James. Complete details can be found in [S1].

Suppose that $Z$ is a separable Banach space and $\Phi(z)$ is defined as in Corollary 9. If $Z^{*}$ is norm separable then it has the Radon-Nikodým property. If $Z^{*}$ is not norm separable then there exists an $f$ as above except that $f$ is only Baire one in the weak* topology and cannot be measurable when $Z$ and $Z^{*}$ have their respective norm topologies, or, even if $Z^{*}$ has its weak topology. See [S1] and its bibliography.

Added in proof. Using the notation of Theorem 5, observe that

$$
\{t \in T: \varrho(\Phi(t), \bar{D})=0\}=\bigcap_{n=1}^{\infty}\left\{t \in T: \Phi(t) \cap\left(\overline{D+B\left(0, \frac{1}{n}\right)}\right) \neq \varnothing\right\} .
$$

The proofs of Theorems 5 and 6 show that the hypotheses of Theorems 5 and 6 may be relaxed by only assuming that

$$
\{t \in T: \varrho(\Phi(t), D)=0\}
$$

is closed for any norm closed, bounded and convex subset $D$ of $X$. This is the hypothesis of Theorem 3. Consider the following quite elementary example. Let $\ell_{1}$ denote the Banach space of absolutely summing sequences with the canonical basis $\left\{e_{n}\right\}$. Define

$$
\begin{aligned}
& C_{0}=\left\{\left(t_{n}\right) \in \ell_{1}: t_{n} \geq 0 \text { for all } n \text { and } \sum_{n} t_{n}=1\right\}, \\
& C_{1}=\left\{\left(\frac{n}{n+1} t_{n}\right) \in \ell_{1}: \sum_{n}\left|t_{n}\right| \leq 1\right\} .
\end{aligned}
$$

Both sets are norm closed, bounded, convex and they are disjoint. Define $T=$ $\left\{1, \ldots, \frac{1}{n}, \ldots, 0\right\}$ and define $\Phi\left(\frac{1}{n}\right)=\left\{\frac{n}{n+1} e_{n}\right\}$ and $\Phi(0)=C_{0}$;

$$
\left\{t: \Phi(t) \cap C_{1} \neq \varnothing\right\}
$$

is not closed. It is easy to see that for any set $D$

$$
\{t \in T: \varrho(\Phi(t), D)=0\}
$$

is either finite or, if it is infinite, then $\varrho(\Phi(0), D)=0$. The interested reader may want to interpret Theorem 5 for hyperspaces in the Vietoris topology and Theorem 6 for hyperspaces in the Hausdorff metric.

\section{BIBLIOGRAPHY}

[B] J. Bourgain, Compact sets of the first Baire class, Bull. Soc. Math. Belgium 29 (1977), 135143.

[BFT] J. Bourgain, D. Fremlin, and M. Talagrand, Pointwise compact sets of Baire measurable functions, Amer. J. Math. 160 (1978), 845-886. 
[Ch] J. P. R. Christensen, Joint continuity of separately continuous functions, Proc. Amer. Math. Soc. 82 (1981), 455-461.

[F] Z. Frolik, A measurable map with analytic domain and metrizable range is quotient, Bull. Amer. Math. Soc. 75 (1970), 1112-1117.

[F2] _ Baire spaces and some generalizations of complete metric spaces, Czech. Math. J. 20 (1970), 406-467.

[H] R. Hansell, First class selectors for upper semi-continuous multifunctions, preprint, 1987.

[HJR] R. Hansell, J. Jayne, and M. Talagrand, First class selectors for weakly upper semi-continuous multi-valued maps in Banach spaces, J. Reine Angew. Math. 361 (1985), 201-220.

[Ha] H. Hausdorff, Mengenlehre (3. Fassung), Dover, New York, 1944.

[JR] J. Jayne and C. A. Rogers, Borel selectors for upper semi-continuous set valued maps, Acta Math. 155 (1985), 41-79.

[K] K. Kuratowski, Topologie, PWN, Warszawa, 1958.

[L] H. Lebesgue, Euvres scientifiques, Volume III, Université de Genève, 1972, 211-217.

[M] Y. N. Moschovakis, Descriptive set theory, North-Holland, Amsterdam, 1980.

[N] I. Namioka, Separate and joint continuity, Pacific J. Math. 51 (1974), 515-531.

[S1] C. Stegall, Applications of descriptive topology in functional analysis, Universität Linz, 1985; 2nd ed., 1987.

[S2] _ Notes, 1976, unpublished.

[S3] _ The duality between Asplund spaces and spaces with the Radon-Nikodym property, Israel J. Math. 29 (1978), 408-412.

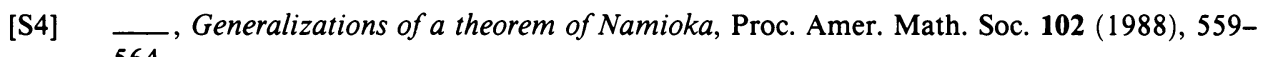
564.

Institut Für Mathematik, JohanNes Kepler Universität, Altenbergerstrasse, A-4040 LINZ, AUSTRIA 